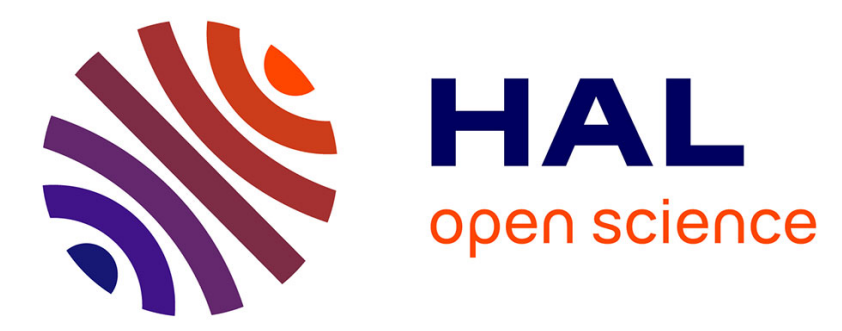

\title{
Evidence of jovian active longitude: 1. Efficiency of cyclotron maser instability.
}

Patrick H. M. Galopeau, Mohammed Y. Boudjada, Helmut O. Rucker

\section{To cite this version:}

Patrick H. M. Galopeau, Mohammed Y. Boudjada, Helmut O. Rucker. Evidence of jovian active longitude: 1. Efficiency of cyclotron maser instability.. Journal of Geophysical Research Space Physics, 2004, 109 (12), pp.A12217. 10.1029/2004JA010459 . hal-00151732

\section{HAL Id: hal-00151732 \\ https://hal.science/hal-00151732}

Submitted on 23 Jan 2016

HAL is a multi-disciplinary open access archive for the deposit and dissemination of scientific research documents, whether they are published or not. The documents may come from teaching and research institutions in France or abroad, or from public or private research centers.
L'archive ouverte pluridisciplinaire HAL, est destinée au dépôt et à la diffusion de documents scientifiques de niveau recherche, publiés ou non, émanant des établissements d'enseignement et de recherche français ou étrangers, des laboratoires publics ou privés. 


\title{
Evidence of jovian active longitude: 1. Efficiency of cyclotron maser instability
}

\author{
Patrick H. M. Galopeau \\ Centre d'Études des Environnements Terrestre et Planétaires, Vélizy, France
}

Mohammed Y. Boudjada and Helmut O. Rucker

Space Research Institute, Austrian Academy of Sciences, Graz, Austria

Received 3 March 2004; revised 13 July 2004; accepted 16 August 2004; published 18 December 2004.

[1] Long-term observations of Jupiter's decametric radiation have shown that a great part of emission is modulated by two dominant factors: the planetary rotation and the orbital phase of Io. The first one indicates that the occurrence probability of the radiation depends on the observer's longitude, while the second factor points to a control of part of the radio emission by Io. Within the framework of the cyclotron maser instability, which is supposed to be the mechanism at the origin of most planetary radio emissions, we estimate the efficiency of this theoretical mechanism at the footprint of the Io flux tube during a complete revolution of the satellite around Jupiter. Our study is based on several simplifying hypotheses: on one hand, we suppose that electrons are accelerated in the neighborhood of Io and follow an adiabatic motion along magnetic field lines carried by the satellite; on the other hand, we assume that a loss cone built up by electrons which have disappeared in Jupiter's ionosphere constitutes the main source of free energy needed by the cyclotron maser instability to produce the radiation. We calculate the maximum growth rate of the waves amplified by the mechanism as a function of the jovicentric longitude of Io. It emerges that some longitudes in the Northern and Southern Hemispheres favor the radio decametric emission and lead to a higher occurrence probability. Our results are compared to the occurrences observed for the sources Io-A, Io-B, Io-C, and Io-D in the usual central meridian longitude-Io phase diagram. INDEX TERMS: 6954 Radio Science: Radio astronomy; 6220 Planetology: Solar System Objects: Jupiter; 6218 Planetology: Solar System Objects: Jovian satellites; 2732 Magnetospheric Physics: Magnetosphere interactions with satellites and rings; 7867 Space Plasma Physics: Wave/particle interactions; KEYWORDS: cyclotron maser instability, Jupiter, planetary radio emissions

Citation: Galopeau, P. H. M., M. Y. Boujada, and H. O. Rucker (2004), Evidence of jovian active longitude: 1. Efficiency of cyclotron maser instability, J. Geophys. Res., 109, A12217, doi:10.1029/2004JA010459.

\section{Introduction}

[2] Jupiter is known to be the most powerful source of nonthermal electromagnetic radiation in the solar system. Its radio spectrum extends from a few $\mathrm{kHz}$ to more than $1 \mathrm{GHz}$. In this paper, we focus on the complex region of Jupiter's spectrum at decameter wavelengths, the so-called DAM. It was discovered by Burke and Franklin [1955] and a number of ground-based observations have systematically monitored this jovian DAM radio source. Several studies [Carr et al., 1983, and references therein] have led to a comprehensive description of the morphological properties of the emissions. The decametric emissions are found to dependent mainly on the following observational parameters: the observation frequency, the jovicentric central meridian longitude (CML), the phase of the satellite Io (hereafter $\Phi_{\mathrm{Io}}$ ), the jovicentric latitude of Earth

Copyright 2004 by the American Geophysical Union. 0148-0227/04/2004JA010459 (hereafter $D_{\mathrm{E}}$ ). These investigations have provided the bases for many models and allowed to specify, e.g., the jovian magnetic field [Connerney, 1993], the coupling of Jupiter's ionosphere and magnetosphere [Smith, 1976], and also the nature of the radio source [Goldstein and Goertz, 1983].

[3] From long-term statistics of the DAM observations, Dulk [1965a, 1965b] proposed a beam model to account for emissions linked to the position of Io in its orbit (the so-called Io-controlled emission). The emission is beamed into a radiation pattern believed to have the form of a hollow cone with apex at the point of emission and axis tangent to the magnetic field direction. This beam model leads to estimate the half-angle of the hollow cone around $80^{\circ}$. The DAM emission is generated in the extraordinary mode (X mode) just above the local cutoff frequency of this mode. The theory of the cyclotron maser instability (CMI) has become relevant as a mechanism responsible for the generation of auroral planetary radio emissions. This mechanism requires electronic distribution functions 
presenting an inversion of population, e.g., loss cone distributions. In the model proposed by $W u$ and Lee [1979] in order to explain the Auroral Kilometric Radiation (AKR), electrons, injected from the plasma sheet and the magnetosphere, are assumed to descend into the ionosphere with varying pitch angles and to create a loss cone distribution function, because of the converging magnetic field lines (magnetic mirror) and the absorption of particles.

[4] In this paper we attempt to prove, in the framework of the CMI theory, the existence of an "active" longitude range rotating with Jupiter and modulating the decametric radio emissions. In section 2 we present the main characteristics of the jovian radio "sources" and the corresponding observational parameters, like the occurrence diagram, the polarization, and the source region. The basic equations which leads to derive the growth rate of the CMI are described in section 3 as well as the loss cone distribution function which serves as a source of free energy. In section 4 we study the theoretical growth rate of the waves as a function of the jovicentric longitude and we deduce a modeled occurrence diagram. Finally, in section 5, we compare both occurrence diagrams, i.e., the modeled and the observed ones, and we discuss their likeness and discrepancy taking into consideration our choice of key parameters.

\section{Observation and Occurrence Probability}

[5] Long term ground observations of the DAM emission reveal that the occurrence probability of the radiation depends on two essential parameters: the central meridian longitude (CML, system III) which is linked to the rotating magnetic field and the orbital phase of the satellite Io [Bigg, 1964]. Several studies [Warwick et al., 1975; Thieman, 1979; Leblanc et al., 1993] were devoted to the analysis of the occurrence probability of the DAM emission over a full jovian period, which is known to be $\approx 12$ years (precisely 4332.589 days). In the CML- $\Phi_{\text {Io }}$ diagram, which describes the DAM occurrence versus CML and $\Phi_{\text {Io, }}$, several zones of enhanced occurrence have been labeled "sources" as displayed in Figure 1. We mainly distinguish four "sources," the so-called Io-controlled emission, i.e., Io-A, Io-B, Io-C and Io-D [Carr and Desch, 1976].

[6] It is difficult to exactly precise the longitude and the Io phase associated to each "source" region. The reason is that the DAM occurrence depends on the geometry EarthSun-Jupiter and the reception system (i.e., antenna and receivers). However it is possible to give, taking into considerations the published catalogues, some estimations of the "source" regions in CML and $\Phi_{\mathrm{Io}}$. According to Carr et al. [1983], the Io-A region is found between $180^{\circ}$ and $300^{\circ}$ in longitude, and in the range $180^{\circ}-260^{\circ}$ with regards to $\Phi_{\mathrm{Io}}$. The maximum frequency of Io-A events is $\approx 38 \mathrm{MHz}$, with dominant right-handed circular (RHC) polarization. The Io-B "source" is observed in the longitude and Io phase intervals, $15^{\circ}-240^{\circ}$ and $40^{\circ}-110^{\circ}$, respectively. The RHC polarization is also the dominant one for Io-B events, with a maximum frequency which could reach $39.5 \mathrm{MHz}$. These two "source" regions are considered to be emitted from the Northern Hemisphere

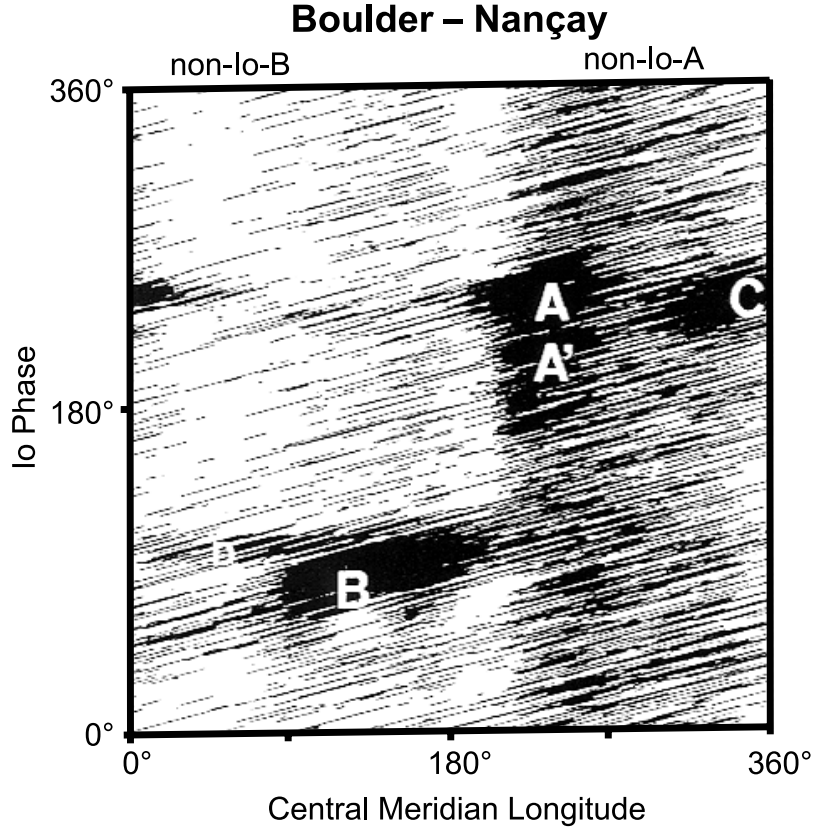

Figure 1. Occurrence probability of jovian decameter radio emissions (as observed by ground-based radio stations) versus system III longitude (CML) and Io phase $\left(\Phi_{\text {Io }}\right)$ [after Genova et al., 1989, and references therein].

because of their senses of polarization [Lecacheux et al., 1991, and references therein]. The Io-C "source" is the only one which exhibits the same amount of both rightand left-handed (LHC) circular polarization. It is mainly observed in the ranges, $280^{\circ}-60^{\circ}$, and, $200^{\circ}-260^{\circ}$, for the CML and $\Phi_{\text {Io }}$, respectively. The last well-known "source," is Io-D, which is nearly observed at the same longitudes as Io-B, between $0^{\circ}$ and $200^{\circ}$, but with a different interval of $\Phi_{\mathrm{Io}}: 95^{\circ}-130^{\circ}$. The emission diagram associated to the DAM "sources" is supposed to be a hollow cone [Dulk, 1965a], where the pairs (Io-B, Io-A) or (Io-C, Io-D) are considered to belong to a same cone which is associated to either the jovian Northern or Southern Hemisphere, respectively. However, recent polarization measurements have shown that RHC and LHC could be observed in all regions [Aubier et al., 2000, and references therein], which means that the observed hollow cone is a superposition of several others coming from both hemispheres.

[7] As shown in Figure 1, we can distinguish the four main "sources" for which we note that the probability of occurrence is important for specific longitudes and Io phases. The occurrence dependence for some specific values of $\Phi_{\text {Io }}$, i.e., $\sim 90^{\circ}$ and $245^{\circ}$, is due to the particular positions of Io in comparison with the observer. The origin of the occurrence dependence in longitudes, i.e., around $90^{\circ}$ and $290^{\circ}$, is still unknown.

[8] In order to interpret the control of the "sources" by the jovian longitude, we have sought whether the mechanism of emission, supposed to be at the origin of DAM, could favor some specific longitudes of Jupiter. With this aim in view, we have calculated the theoretical growth rate of the waves as a function of the jovicentric longitude and we have made the assumption that the greater this growth 
rate is, the greater the occurrence probability of the radio emission should be.

\section{Growth Rate of the Waves in the Framework of the Cyclotron Maser Instability}

\subsection{Mechanism of Emission}

[9] The generation of planetary radio emissions (in particular the Earth auroral kilometric radiation, AKR, and jovian decametric emissions, DAM) was first attributed to a Doppler-shifted cyclotron resonance instability [Ellis, 1965; Melrose, 1976], and later on to the cyclotron maser instability (CMI) theory introduced by Wu and Lee [1979]. In their paper, the latter show that their theory differs from the previous ones in two important respects: (1) the role of mirror-reflected auroral electrons whereas other models involve precipitating electrons, and (2) a direct amplification of electromagnetic waves on extraordinary and ordinary mode while the previous theories relied on multistep processes. Such a direct excitation mechanism, based on a relativistic gyroresonance, had been considered by $W u$ and Freund [1977] to study the generation of the Io-modulated component of the jovian decametric radiation and two years after the CMI was first proposed by Wu and Lee [1979] to explain the AKR emission. These authors showed that a kinetic instability exists when a population of suprathermal electrons possesses a loss cone distribution function [Lee and Wu, 1980; Wu et al., 1982]. This instability process causes coherent wave growth near the electron cyclotron frequency.

[10] More generally, the cyclotron maser instability is a resonant coupling between right-handed electromagnetic waves (relatively to the local magnetic field) and an electron population forming a magnetized plasma. The source of free energy needed by the CMI is contained in a positive gradient in perpendicular velocity $v_{\perp}$ of the electron distribution function $f$; i.e., $\partial F / \partial v_{\perp}$ must be positive in certain domains of the momentum space [Le Quéau et al., 1984a, 1984b; Ladreiter, 1991; Omidi and Gurnett, 1982; Omidi et al., 1984]. Finally, a strongly polarized X mode radiation escapes from the source region. It has been verified that the waves originate primarily in the extraordinary wave mode, and near the cyclotron frequency, for AKR [Hanasz et al., 2000, and references therein] and DAM emissions [Lecacheux et al., 1991, and references therein].

\subsection{Hypotheses}

[11] With the intent to determine the maximum growth rate of the waves produced by the CMI, we have ventured several simplifying hypotheses. We have supposed that the radiation generated by the $\mathrm{CMI}$ is emitted within a hollow cone at the local gyrofrequency, the source of free energy needed by the CMI is a loss cone built up by electrons which disappear in Jupiter's ionosphere. We have also assumed that these electrons, accelerated in the vicinity of Io, follow an adiabatic motion along the magnetic field lines linked to Io. When they penetrate into the jovian ionosphere, their probability of disappearance is assumed to be proportional to the length of their actual path and to the ionosphere density. We make the assumption that the distribution function of the electrons responsible for the radiation is Maxwellian at Io. Finally, taking into consideration the previous set of hypotheses, we derive the maximum growth rate $\omega_{i}$ of the CMI as a function of the jovian longitude.

\subsection{Resonance Requirements}

[12] A condition for the resonance, inferred by the CMI, between the electron population and the growing wave to be efficient and lead to a strong amplification is that the electrons in the medium must interact with the electric field of the unstable wave during a large number of periods. Now the wave-particle resonance may go out of tune because of the spatial variability of the magnetic field, the characteristic length scale of which is $L_{B} \sim B /\|\nabla B\|$. In the case of Jupiter, according to the various magnetic field models and in particular $\mathrm{O}_{6}$ proposed by Connerney [1992, 1993], the order of magnitude of the magnetic field at the "surface" and its gradient are respectively: $B \sim 10 \mathrm{G}$ and $\|\nabla B\| \sim$ $40 \mathrm{GR}_{\mathrm{J}}^{-1}$ so that $L_{B} \sim 0.25 R_{\mathrm{J}} \simeq 18,000 \mathrm{~km}$ which is much greater than the wavelengths $\lambda_{\mathrm{DAM}} \sim 15 \mathrm{~m}$ (at $20 \mathrm{MHz}$ ) considered for the DAM emission. Moreover, in the source region, $L_{B}$ is roughly proportional to the radial distance (from the center of Jupiter) so that the proposition $\lambda_{\text {DAM }} \ll L_{B}$ is always fulfilled. As a consequence, the theory of CMI in a homogeneous medium is adapted to the case of the DAM waves generation. In Appendix A, we present a detailed calculation, inspired by [Le Quéau et al., 1984a, 1984b], of the linear growth rate of the waves generated by the CMI in a homogeneous medium. The general equation of dispersion for a right-handed mode is:

$$
k^{2}-\frac{\omega^{2}}{c^{2}}-\frac{\mu_{0} e^{2}}{4 \pi m} \omega \iint \frac{\partial f / \partial v_{\perp}}{\omega-k_{\|} v_{\|}-\omega_{c} / \gamma} v_{\perp}^{2} d v_{\perp} d v_{\|}=0
$$

where $\mathbf{k}$ and $\omega$ refer to the wave number and the frequency of the wave, $v_{\perp}$ and $v_{\|}$are the components of the electron velocity perpendicular and parallel relatively to the ambient magnetic field, $\omega_{c}$ denotes the local gyrofrequency, $\gamma$ the Lorentz factor and $m$ the electron mass. The frequency is a complex parameter:

$$
\omega=\omega_{r}+i \omega_{i}, \quad \omega \in \mathbf{C}, \quad \omega_{r}, \omega_{i} \in \mathbf{R} .
$$

[13] The growth rate $\omega_{i}$ (imaginary part of the frequency) can be derived from equation (1a), $\omega_{p}$ denoting the plasma frequency:

$$
\omega_{i}=\frac{\omega_{p}^{2}}{8} \int_{0}^{+\infty} v_{\perp}^{2} d v_{\perp} \int_{-\infty}^{+\infty} \frac{\partial f}{\partial v_{\perp}} \delta\left(\omega_{r}-k_{\|} v_{\|}-\frac{\omega_{c}}{\gamma}\right) d v_{\|}
$$

with the following normalization:

$$
\iiint f\left(v_{\|}, v_{\perp}\right) d^{3} \mathbf{v}=1 .
$$

The Dirac function in equation (2a) is a resonant condition in the momentum space and the growth rate is an integral along this resonance condition.

\subsection{Distribution Function}

[14] We consider two distinct positions, at abscissa $s$ and $s^{\prime}$, upon a magnetic field line carried away by Io. We assume 
that there is no collision along the magnetic field line so that the number of electrons is retained:

$f(s, v, \alpha) v^{2} \sin \alpha d \alpha d v d s d S=f\left(s^{\prime}, v^{\prime}, \alpha^{\prime}\right) v^{\prime 2} \sin \alpha^{\prime} d \alpha^{\prime} d v^{\prime} d s^{\prime} d S^{\prime}$

$\alpha$ is the pitch angle, $d S$ the infinitesimal cross section of an elementary flux tube and $d s$ the movement along the field line (see Figure 2).

[15] The magnetic flux is always constant:

$$
B \cdot d S=B^{\prime} \cdot d S^{\prime}
$$

[16] The motion is supposed to be adiabatic:

$$
\frac{v^{2} \sin ^{2} \alpha}{B}=\frac{v^{\prime 2} \sin ^{2} \alpha^{\prime}}{B^{\prime}}
$$

and the kinetic energy is constant, so that:

$$
v=v^{\prime}
$$

Then, we obtain:

$$
\frac{d s}{\cos \alpha}=\frac{d s^{\prime}}{\cos \alpha^{\prime}}
$$

[17] Replacing therefore equations (4)-(7) in equation (3) we notice that the distribution function along the magnetic field line is constant (in the position-momentum space):

$$
f(s, v, \alpha)=f\left(s^{\prime}, v^{\prime}, \alpha^{\prime}\right) .
$$

\subsection{Probability of Collision}

[18] The actual path $d l$ of the electrons (with pitch angle $\alpha$ ) during their adiabatic motion corresponding to a parallel movement $d s$ is:

$$
d l= \pm \frac{d s}{\cos \alpha}
$$

We suppose that the probability of collision $\mathcal{P}$ between an individual electron and the ions in the jovian ionosphere is

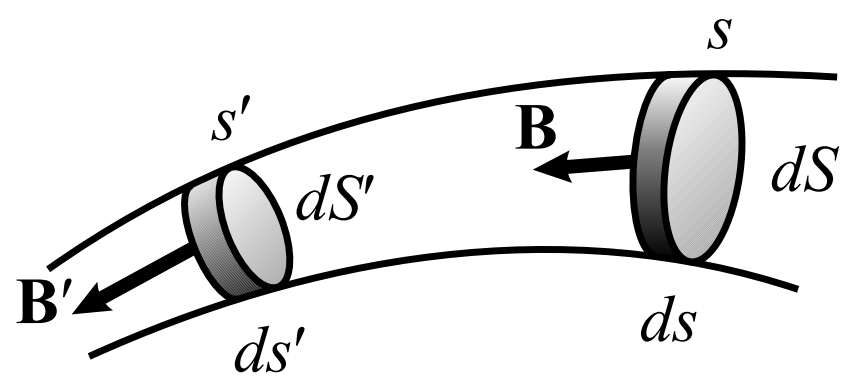

Figure 2. Diagram of an elementary flux tube. Each position along the tube is described by the curvilinear abscissa $s, \mathbf{B}$ is the magnetic field, $d S$ the section of the flux tube, and $d s$ an elementary movement along the tube. Two distinct positions are displayed here for abscissa $s$ and $s^{\prime}$.

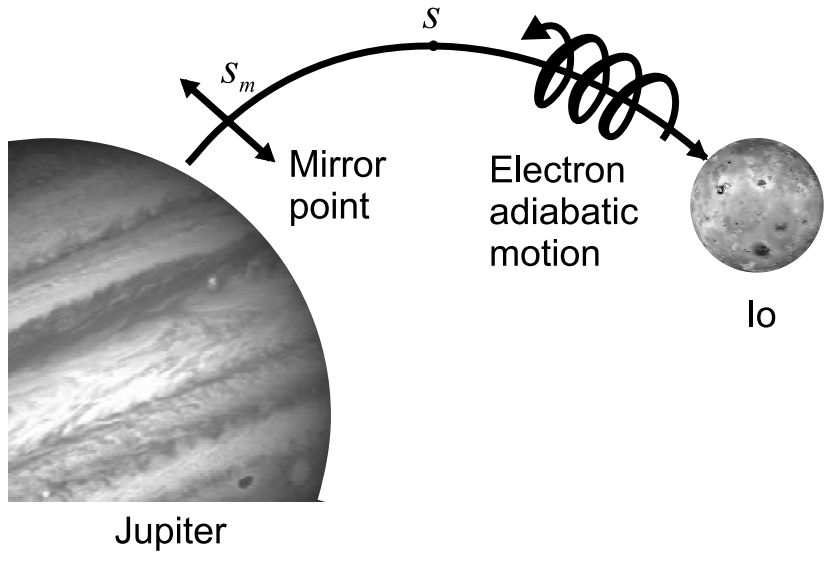

Figure 3. In our scenario, electrons are supposed to follow an adiabatic motion along magnetic field lines from Io toward Jupiter and then to be reflected at their mirror point the abscissa of which is $s_{m}$.

directly proportional to the path followed by the electron and to the density of ions:

$$
d \mathcal{P}=C \cdot e^{-z / H} \cdot d l,
$$

supposing an exponential decay for the ions density characterized by a scale height $H$. $C$ is a constant depending on the cross section of collision and $z$ the jovian altitude.

[19] The whole probability $\mathcal{P}$ is derived from an integration of equation (10) along the full field line from Io $(+\infty)$ to the mirror point $\left(s_{m}\right.$, see Figure 3$)$ and then from the mirror point $\left(s_{m}\right)$ to the current position $(s)$ :

$$
\mathcal{P}=\int_{+\infty}^{s_{m}} \frac{d \mathcal{P}(s)}{d s} d s+\int_{s_{m}}^{s} \frac{d \mathcal{P}(s)}{d s} d s .
$$

Neglecting the probability of collision for electrons between Io and the current position and taking into account equations (9) and (10), we get:

$$
\mathcal{P}=2 C \int_{s_{m}}^{+\infty} e^{-z / H} \frac{d s}{\cos \alpha} .
$$

[20] The density of ions is a steeply decreasing function of the altitude $z$, then collisions are the most efficient near the level $z=0$. On the other hand, the relative decrease of the magnetic field, as a function of $z$, is smaller than that of the density; for this reason, we use a (first-order) linear expansion of $B: B(s)=B_{s}-g_{\|} s$ and we assimilate the magnetic field line near $z=0$ to a portion of straight line (see Figure $4, g_{\|}=\nabla_{\|} B$ denotes the parallel magnetic field gradient and $B_{s}$ the magnetic field strength at level 0). As a consequence, we may write:

$$
z=s \cdot \sin \lambda,
$$

where $\lambda$ is the inclination angle of the field line. Then from equations (5) and (6) we can get $\cos \alpha$ easily:

$$
\cos \alpha=\sqrt{1-\frac{B(s)}{B_{m}}}=\sqrt{1-\frac{B_{s}-g_{\|} \cdot s}{B_{s}-g_{\|} \cdot s_{m}}},
$$




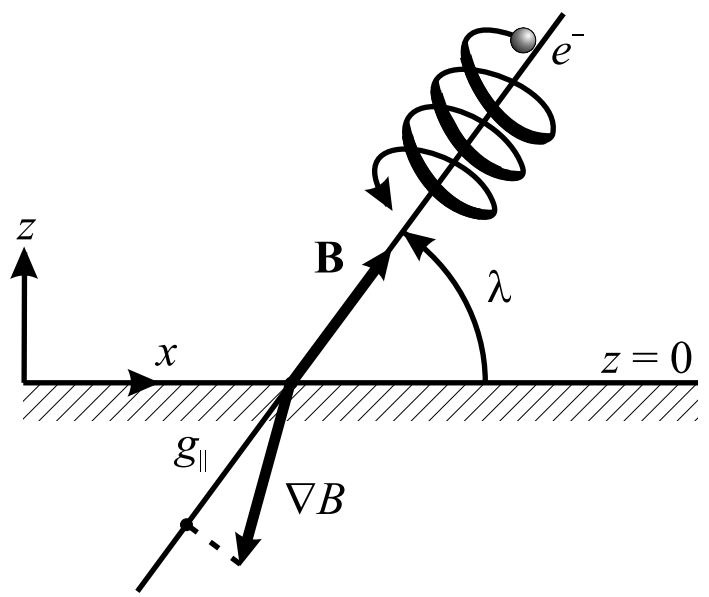

Figure 4. Magnetic field line, in the vicinity of altitude $z=0$ in the ionosphere. This part of the line is considered to be rectilinear. Here $g_{\|}$denotes the parallel component of $\nabla B$, and $\lambda$ is the inclination of $\mathbf{B}$ at altitude $z=0$.

$B_{m}$ denotes the magnetic field strength at the mirror point. In the end, after integration of equation (12), we derive the probability of collision in the ionosphere for an upgoing electron as a function of the pitch angle $\alpha$ and the current abscissa $s$ :

$$
\mathcal{P}(\alpha, s)=2 \sqrt{\pi} C \sqrt{\frac{H \cdot B(s)}{g_{\|} \sin \lambda \sin ^{2} \alpha}} \exp \left[-\frac{\sin \lambda}{H g_{\|}}\left(B_{s}-\frac{B(s)}{\sin ^{2} \alpha}\right)\right] .
$$

[21] In all calculations, we use the jovian magnetic field model $\mathrm{O}_{6}$ proposed by Connerney [1992, 1993]. This model is based mainly on Pioneer 11 and Voyager 1 data and it is supposed to present the best agreement with observations of UV aurora and the Io flux tube location. In Table 1 we list the position of the Io flux tube footprint, the components of the magnetic field vector $\mathbf{B}$, and those of the gradient of its modulus $\nabla B$ as a function of the jovicentric longitude of Io with a step of $30^{\circ}$. As discussed in the beginning of section 3.3, one can verify that the characteristic length scale $L_{B} \sim B /\|\nabla B\|$ is much greater than decameter wavelengths for all longitudes of Io. With regards to the scale height of ionosphere, we have chosen $H=960 \mathrm{~km} \simeq 0.013 R_{\mathrm{J}}$ (with $1 R_{\mathrm{J}}=71,398 \mathrm{~km}$ ) in order to be consistent with Voyager observations [Eshleman et al., 1979a, 1979b].

[22] In other respects, the determination of the constant $C$ is much more delicate: it must include the cross sections of collision of an electron with every species (ions and neutrals) present in the jovian ionosphere at different altitudes. In the present work, we only need the order of magnitude of $C$. Consequently, we have decided to choose $C$ in order to have $\mathcal{P}=1$ for electrons having their mirror point at the place where $B_{s}$ is maximum in the Northern Hemisphere. This is achieved when the jovicentric longitude of Io is equal to $124^{\circ}$. In this case, we have:

$$
C=\frac{1}{2 \sqrt{\pi}} \sqrt{\frac{g_{\|} \sin \alpha}{H B_{s}}}=4.74 R_{\mathrm{J}}^{-1},
$$

Table 1. Components of the Magnetic Field and Gradient of Its Modulus at the Footprint of the Io Flux Tube for Different Values of Io's

\begin{tabular}{|c|c|c|c|c|c|c|c|c|c|}
\hline $\begin{array}{l}\text { Longitude } \\
\text { of Io, deg }\end{array}$ & $\begin{array}{c}\text { Footprint } \\
\text { Longitude, deg }\end{array}$ & $\begin{array}{c}\text { Footprint } \\
\text { Latitude, deg }\end{array}$ & $\begin{array}{c}\text { B Field } \\
\text { Strength G }\end{array}$ & $\begin{array}{l}\text { Colatitude } \\
\text { of } \mathbf{B}, \text { deg }\end{array}$ & $\begin{array}{l}\text { Azimuth } \\
\text { of } \mathbf{B}, \text { deg }\end{array}$ & $\begin{array}{c}\nabla B \text { Strength } \\
\mathrm{G} \cdot R_{\mathrm{J}}^{-1} \\
\end{array}$ & $\begin{array}{l}\text { Colatitude } \\
\text { of } \nabla \mathbf{B}, \text { deg }\end{array}$ & $\begin{array}{c}\text { Azimuth of } \\
\nabla B, \operatorname{deg}\end{array}$ & $\begin{array}{c}\text { Angle } \\
(\mathbf{B}, \nabla B), \text { deg }\end{array}$ \\
\hline \multicolumn{10}{|c|}{ Northern Hemisphere } \\
\hline 0 & 12.51 & 80.95 & 10.20 & 22.17 & 147.60 & 35.25 & 162.39 & 319.03 & 174.60 \\
\hline 30 & 66.45 & 77.53 & 11.32 & 18.86 & 117.46 & 40.38 & 165.96 & 285.06 & 174.07 \\
\hline 60 & 101.91 & 71.28 & 12.58 & 15.21 & 108.57 & 46.05 & 169.82 & 272.20 & 173.86 \\
\hline 90 & 126.12 & 63.91 & 13.56 & 11.49 & 117.07 & 50.39 & 173.76 & 277.77 & 174.03 \\
\hline 120 & 144.38 & 57.29 & 13.97 & 8.74 & 143.67 & 52.06 & 176.80 & 317.59 & 174.44 \\
\hline 150 & 159.95 & 52.96 & 13.78 & 8.70 & 184.63 & 51.10 & 175.74 & 29.72 & 174.84 \\
\hline 180 & 174.71 & 51.78 & 13.13 & 12.09 & 216.86 & 48.15 & 171.79 & 55.88 & 174.92 \\
\hline 210 & 189.86 & 54.12 & 12.16 & 17.40 & 233.29 & 43.82 & 167.06 & 65.17 & 174.59 \\
\hline 240 & 207.08 & 59.83 & 11.01 & 22.95 & 238.71 & 38.74 & 162.16 & 66.19 & 174.27 \\
\hline 270 & 229.55 & 67.63 & 9.94 & 26.82 & 233.96 & 33.99 & 158.30 & 57.95 & 174.63 \\
\hline 300 & 262.05 & 74.98 & 9.35 & 27.35 & 217.87 & 31.31 & 157.24 & 37.85 & 175.41 \\
\hline 330 & 309.54 & 79.91 & 9.47 & 25.21 & 188.97 & 31.88 & 159.17 & 4.50 & 175.28 \\
\hline \multicolumn{10}{|c|}{ Southern Hemisphere } \\
\hline 0 & 3.89 & -64.08 & 9.17 & 160.21 & 168.32 & 30.24 & 166.11 & 151.40 & 7.62 \\
\hline 30 & 35.00 & -63.64 & 8.48 & 157.08 & 180.05 & 26.11 & 162.06 & 168.88 & 6.31 \\
\hline 60 & 65.03 & -61.15 & 8.25 & 158.15 & 197.62 & 24.11 & 162.31 & 197.50 & 4.16 \\
\hline 90 & 88.14 & -59.49 & 8.67 & 162.81 & 203.90 & 25.80 & 166.37 & 216.89 & 4.94 \\
\hline 120 & 108.52 & -61.52 & 9.38 & 167.26 & 195.81 & 28.92 & 170.71 & 219.98 & 5.69 \\
\hline 150 & 132.04 & -66.56 & 10.06 & 169.64 & 179.65 & 31.96 & 173.80 & 209.45 & 5.85 \\
\hline 180 & 164.86 & -71.87 & 10.51 & 169.84 & 168.85 & 34.34 & 175.11 & 191.20 & 5.94 \\
\hline 210 & 207.79 & -74.09 & 10.68 & 168.56 & 172.53 & 35.70 & 174.63 & 182.40 & 6.22 \\
\hline 240 & 249.12 & -72.74 & 10.64 & 166.91 & 181.20 & 36.23 & 173.60 & 185.10 & 6.72 \\
\hline 270 & 283.10 & -69.78 & 10.50 & 165.89 & 185.06 & 36.27 & 173.15 & 183.99 & 7.27 \\
\hline 300 & 311.54 & -66.64 & 10.25 & 165.30 & 180.85 & 35.57 & 172.70 & 170.53 & 7.62 \\
\hline 330 & 337.13 & -64.54 & 9.81 & 163.67 & 171.45 & 33.64 & 170.43 & 153.71 & 7.77 \\
\hline
\end{tabular}
Longitude Around Jupiter ${ }^{\mathrm{a}}$

${ }^{a}$ The magnetic field model used here is $\mathrm{O}_{6}$ by Connerney [1992, 1993]. The columns give respectively: the jovicentric longitude of Io, the longitude and latitude of the footprint of the field line connected to Io, the strength, the colatitude and the azimuth of $\mathbf{B}$, the strength, the colatitude and the azimuth of $\nabla B$, and finally the angle between $\mathbf{B}$ and $\nabla B$. The colatitude is computed from the north pole and the azimuth corresponds to the clockwise angle between the direction of the north pole and the tangential component of $\mathbf{B}$ or $\nabla B$. 

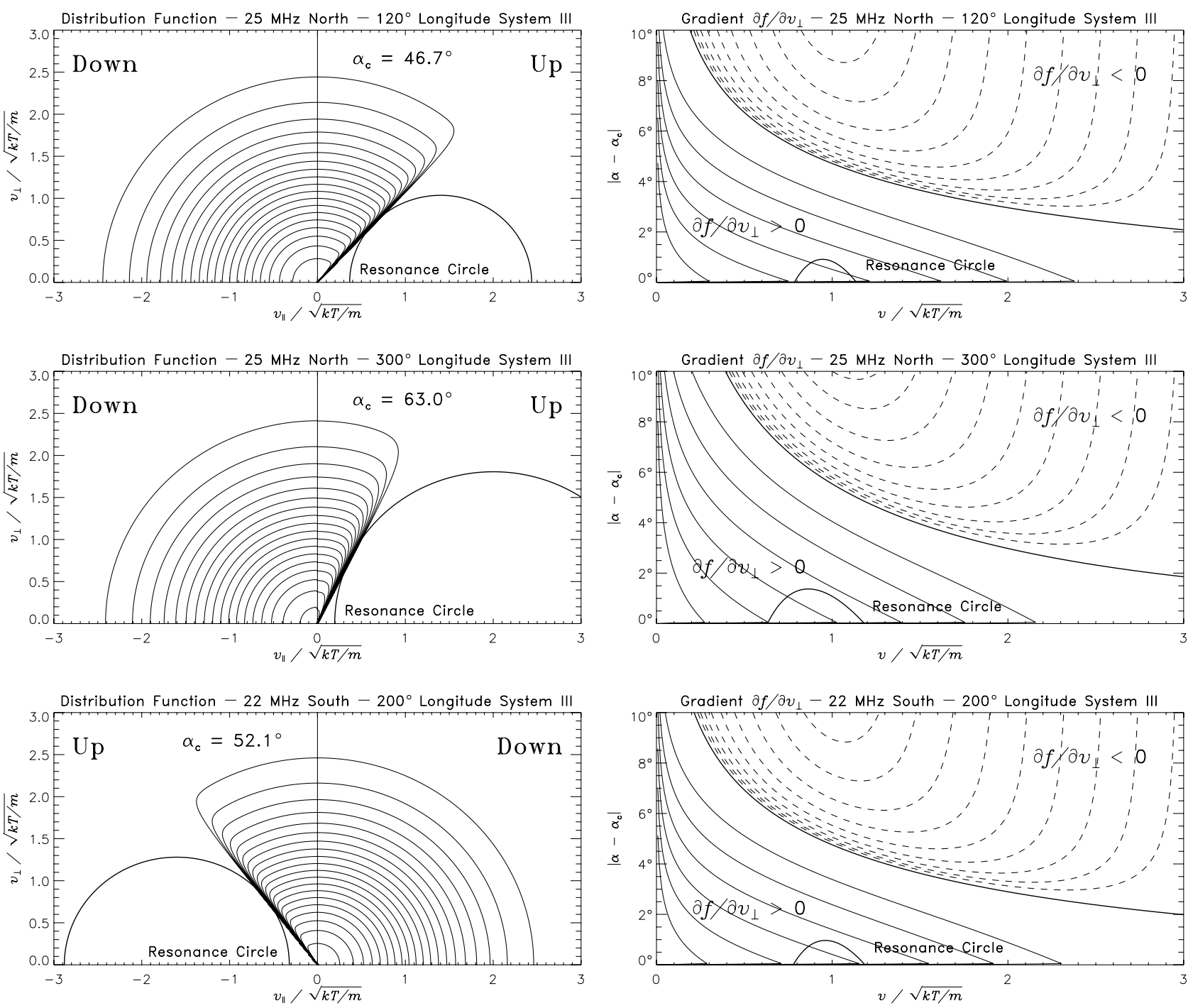

Figure 5. Left panels show contours of the electron distribution function $f$ at the point of the magnetic field line where the gyrofrequency is $25 \mathrm{MHz}$ (Northern Hemisphere) or $22 \mathrm{MHz}$ (Southern Hemisphere). It is plotted for three different longitudes (of system III): $120^{\circ}, 300^{\circ}$ (north) and $200^{\circ}$ (south). Right panels show contours of the perpendicular gradient of the distribution function $\partial f / \partial v_{\perp}$ near the edge of the loss cone plotted for the same values of gyrofrequencies and longitudes. The loss cone is characterized by its opening angle $\alpha_{c}$. The optimum resonance circle defined in subsection 3.7 is also displayed; it is tangential to the edge of the loss cone and only a very small portion of the circle penetrates into the region where $\partial f / \partial v_{\perp}>0$. In all the plots the velocity is normalized to $\sqrt{k T / m}$ where $T$ is the temperature of the initial Maxwellian distribution function at Io. Here $k$ refers to Boltzmann's constant.

with the following values at the footprint of the Io flux tube: $B_{s}=13.97 \mathrm{G}, \lambda=81^{\circ} 28^{\prime}$ and $g_{\|}=51.84 \mathrm{G} / R_{\mathrm{J}}$ derived from the $\mathrm{O}_{6}$ model.

\subsection{Growth Rate}

[23] Supposing that the electron distribution function at Io is a Maxwellian characterized by a temperature $T$, we can derive from equations (8) and (15) the distribution function $f(s, \alpha, v)$ at any position $s$ upon the magnetic field line carried away by Io. So, in the case of down-going electrons toward Jupiter, we get:

$$
f_{\text {down }}(s, \alpha, v)=\left(\frac{m}{2 \pi k T}\right)^{3 / 2} e^{-m v^{2} / 2 k T}
$$

for pitch angles in the interval $\pi / 2 \leq \alpha \leq \pi$ for the Northern Hemisphere or $0 \leq \alpha \leq \pi / 2$ for the Southern Hemisphere. Concerning upgoing electrons (toward Io), a part of them has disappeared in the jovian ionosphere so that the distribution function is:

$$
f_{\text {up }}(s, \alpha, v)=[1-\mathcal{P}(s, \alpha)]\left(\frac{m}{2 \pi k T}\right)^{3 / 2} \frac{e^{-m v^{2} / 2 k T}}{\cos ^{2} \frac{\alpha_{c}}{2}}
$$

where $\alpha_{c}$ denotes the angle of the loss cone and corresponds to $\mathcal{P}\left(s, \alpha_{c}\right)=1$. This equation applies to the situation $\alpha_{c} \leq \alpha \leq \pi / 2$ (north) or $\pi / 2 \leq \alpha \leq \pi-\alpha_{c}$ (south). Everywhere else $f_{\text {up }}(s, \alpha, v)=0$. The contours of this distribution function are plotted in Figure 5 (left 


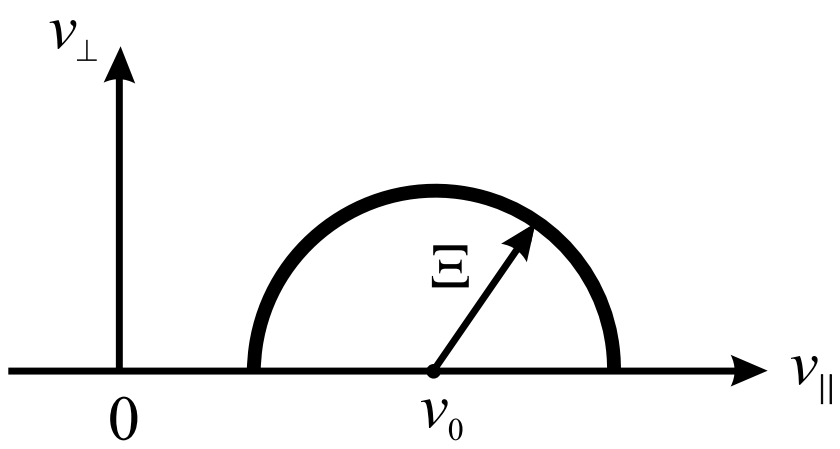

Figure 6. Resonance curve of the cyclotron maser instability in velocity space $\left(v_{\|}, v_{\perp}\right)$ : it is approximated to a circle of radius $\Xi$.

panels) for three distinct longitudes of Io: $120^{\circ}$ and $300^{\circ}$ at a position corresponding to $25 \mathrm{MHz}$ (in the Northern Hemisphere) and $200^{\circ}$ at $22 \mathrm{MHz}$ (in the Southern Hemisphere).

[24] The perpendicular gradient is easily obtained:

$$
G_{\perp}=\frac{\partial f}{\partial v_{\perp}}=\frac{\cos \alpha}{v} \frac{\partial f}{\partial \alpha}+\sin \alpha \frac{\partial f}{\partial v} .
$$

Contours of $G_{\perp}$ are plotted in Figure 5 (right panels) for the same longitudes of Io as a function of $v$ and $\left|\alpha-\alpha_{c}\right|$. It is worth noticing that the perpendicular gradient $G_{\perp}$ is positive only in a very thin region near the edge of the loss cone (of angle $\alpha_{c}$ ).

[25] In the end, replacing equation (18) in equation (2a), it is possible to derive the maximum growth rate $\omega_{i}$ of the CMI from an integration along the resonance curve which can be approximated to a circle in the plane $\left(v_{\|}, v_{\perp}\right)$ :

$$
\omega_{i}=\frac{\omega_{p}^{2} c^{2}}{8 \omega_{c}} \Xi^{2} \int_{0}^{\pi} G_{\perp}(\Xi, \theta) \sin ^{2} \theta d \theta .
$$

The radius of the resonance circle is:

$$
\Xi=c\left[\frac{k_{\|}^{2} c^{2}}{\omega_{c}^{2}}+2\left(1-\frac{\omega_{r}}{\omega_{c}}\right)\right]^{1 / 2}
$$

and its center is positioned at the point $v_{\perp}=0, v_{\|}=v_{0}$ (see Figure 6) with:

$$
v_{0}=\frac{k_{\|} c^{2}}{\omega_{c}^{2}} .
$$

\subsection{Optimum Resonance Circle}

[26] We assume that the waves having a strong occurrence probability are those produced with a maximum growth rate. We numerically determine, at a given frequency of emission $(25 \mathrm{MHz}$ or $22 \mathrm{MHz}$, for the radiation escaping from the Northern or Southern Hemisphere, respectively), the position of the resonance circle $\left(v_{0}, \Xi\right)$ for which the growth rate $\omega_{i}$ is positive and maximum, for each position of the magnetic field line intersecting Io and characterized by its longitude. As displayed in Figure 5, the particular resonance circle leading to this maximum growth rate is nearly tangent to the edge of the loss cone of the distribution function and penetrates just in the region where $\partial f / \partial v_{\perp}>0$.

\section{Results}

\subsection{Growth Rate Versus Jovian Longitude}

[27] In Figure 7, the maximum growth rate (actually a dimensionless normalized growth rate $\omega_{i} \omega_{c} k T / \omega_{p 0}^{2} m c^{2}$ where $\omega_{p 0}$ is the plasma frequency at Io) is plotted as a function of the jovian longitude of the magnetic field line along which the radio emission is supposed to occur. The calculation has been made for several frequencies: 25, 20, 15 and $12 \mathrm{MHz}$ for the Northern Hemisphere and 22, 18, 15 and $12 \mathrm{MHz}$ for the Southern Hemisphere. We note that the CMI growth rate is quasi-constant and maximal at low frequency $(\sim 12 \mathrm{MHz})$. Toward higher frequencies, the growth rate curves present a longitude modulation with two extreme points which correspond to the maximum and minimum efficiency of the CMI mechanism. This modulation in longitude allows to define the extent of the efficiency domain. It is very pronounced beyond $20 \mathrm{MHz}$ but fades very quickly when the frequency decreases and practically disappears below $12 \mathrm{MHz}$.

[28] With regard to the DAM radiation coming from the Northern and Southern Hemispheres, the maxima of the CMI efficiency are around $130^{\circ}$ and $200^{\circ}$, respectively. These values can be interpreted as the location of an active longitude where the emission mechanism is the most effective. Figure 7 also suggests that the CMI mechanism might be more efficient in the Northern Hemisphere as greater values of $\omega_{i}$ are attained.

\subsection{Source Location}

[29] Figure 8 displays the contours of the angle between the direction of the observer (located at Earth) and the local magnetic field, at the place where the emission occurs. The abscissa is the jovicentric longitude (system III) of Earth and the ordinate is the longitude of Io. These contours are considered for two representative frequencies $(25 \mathrm{MHz}$ and $22 \mathrm{MHz}$ for the Northern and Southern Hemispheres, respectively) and a constant jovicentric declination $D_{\mathrm{E}}$ equal to $0^{\circ}$.

[30] The grey area corresponds to a certain domain of active longitude, carried away by Jupiter, and deduced from the curves in Figure 7. Two conditions are needed for the radio emission to be observed at Earth's orbit: (1) the magnetic field line connected to Io must cross the CMI active domain linked to Jupiter, and in the same time (2) the observer-Jupiter line of sight must be included in the hollow cone representing the beaming of the radiation. The first condition is needed by the CMI to be efficient and corresponds to the grey area displayed in Figure 8. The second condition is fulfilled by supposing that the emission occurs symmetrically around the direction of the magnetic field, in a hollow cone of a constant opening angle. In the case of our study, we consider two opening angles equal to $70^{\circ}$ and $110^{\circ}$ for the Northern and the Southern Hemispheres, respectively. These values are deduced from previous results [see Leblanc et al., 1994, 

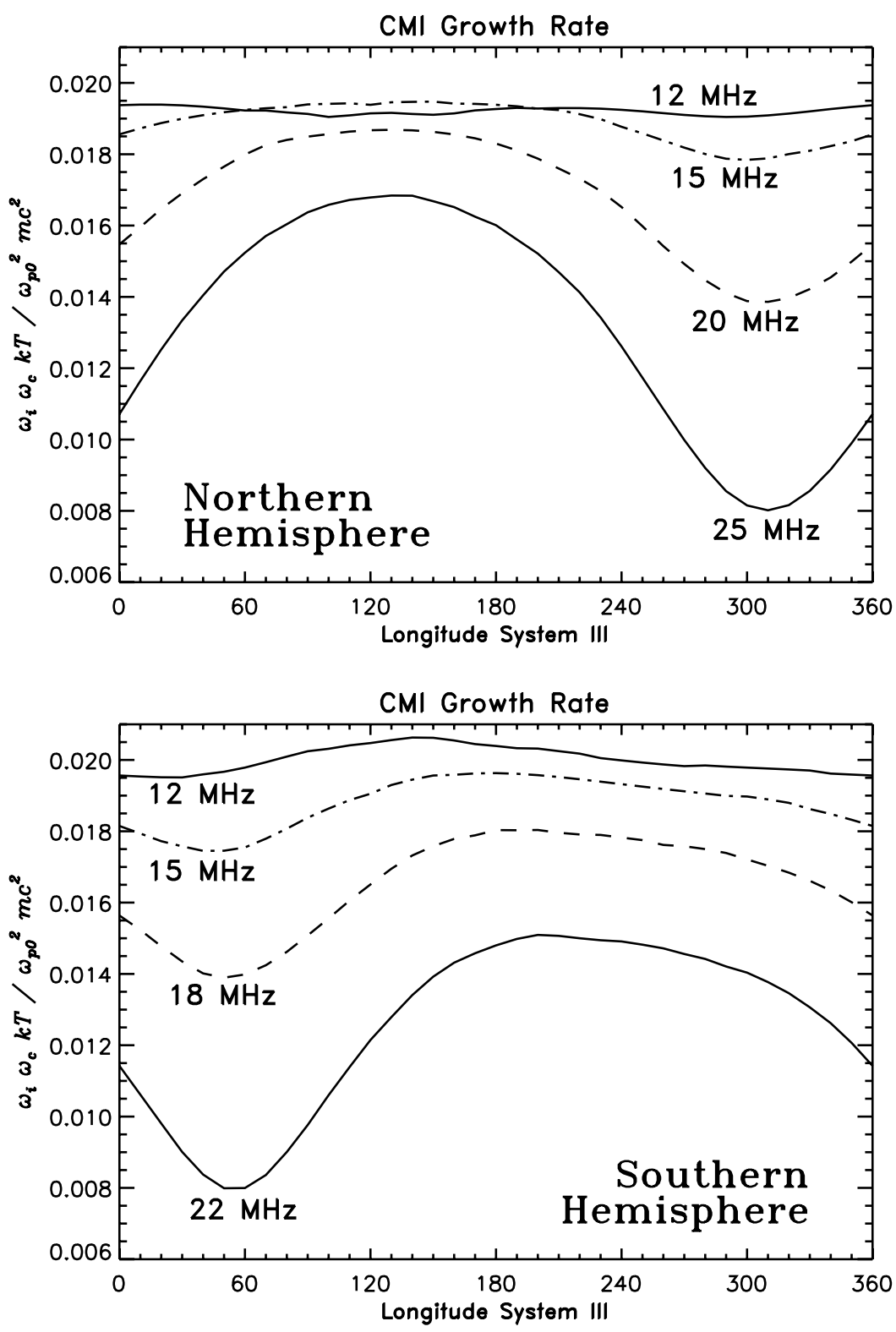

Figure 7. Dimensionless normalized growth rate of the cyclotron maser instability versus jovian longitude (system III), calculated for both hemispheres for four frequencies: 25, 20, 15, $12 \mathrm{MHz}$ (north) or $22,18,15,12 \mathrm{MHz}$ (south). The growth rate $\omega_{i}$ is normalized to $\omega_{p 0}^{2} m c^{2} / \omega_{c} k T$, where $\omega_{p 0}^{2}$ and $T$ are the plasma frequency and the temperature of the initial electron distribution function at Io and $\omega_{c}$ the local gyrofrequency.

and references therein] for the Io-controlled "source" regions associated to the Northern and Southern Hemispheres. Thus the two conditions required for the emission are simultaneously fulfilled for the segments of the contours $70^{\circ}$ (or $110^{\circ}$ ) which are located in the grey areas and marked by thick lines. For each hemisphere we get two segments corresponding to two edges of a same hollow cone.

[31] By examining Figure 8, one can notice that, on the one hand, segments B and D can be simultaneously observed for Earth's longitudes ranging from $50^{\circ}$ to $130^{\circ}$, and segments A and C for Earth's longitudes between $180^{\circ}$ and $240^{\circ}$. On the other hand, the four segments correspond to a common interval of Io's longitude ranging from $120^{\circ}$ to $200^{\circ}$. These overlaps can make more difficult the distinction between the different "source" regions.

\subsection{CML-Io Phase Diagram}

[32] In order to compare the theoretical zones of high emission probability with the observations, in particular with the occurrence diagram (see Figure 1), it is relevant to use a representation involving the phase of Io rather than its longitude. Thus in Figure 9, the theoretical "sources" are displayed in a usual CML-Io phase diagram. In the following we analyze the distribution of such "sources," for a fixed jovicentric declination equal to $0^{\circ}$, taking into consideration three fundamental parameters: the central meridian longitude, the orbital phase of Io $\Phi_{\text {Io }}$ and the observed 

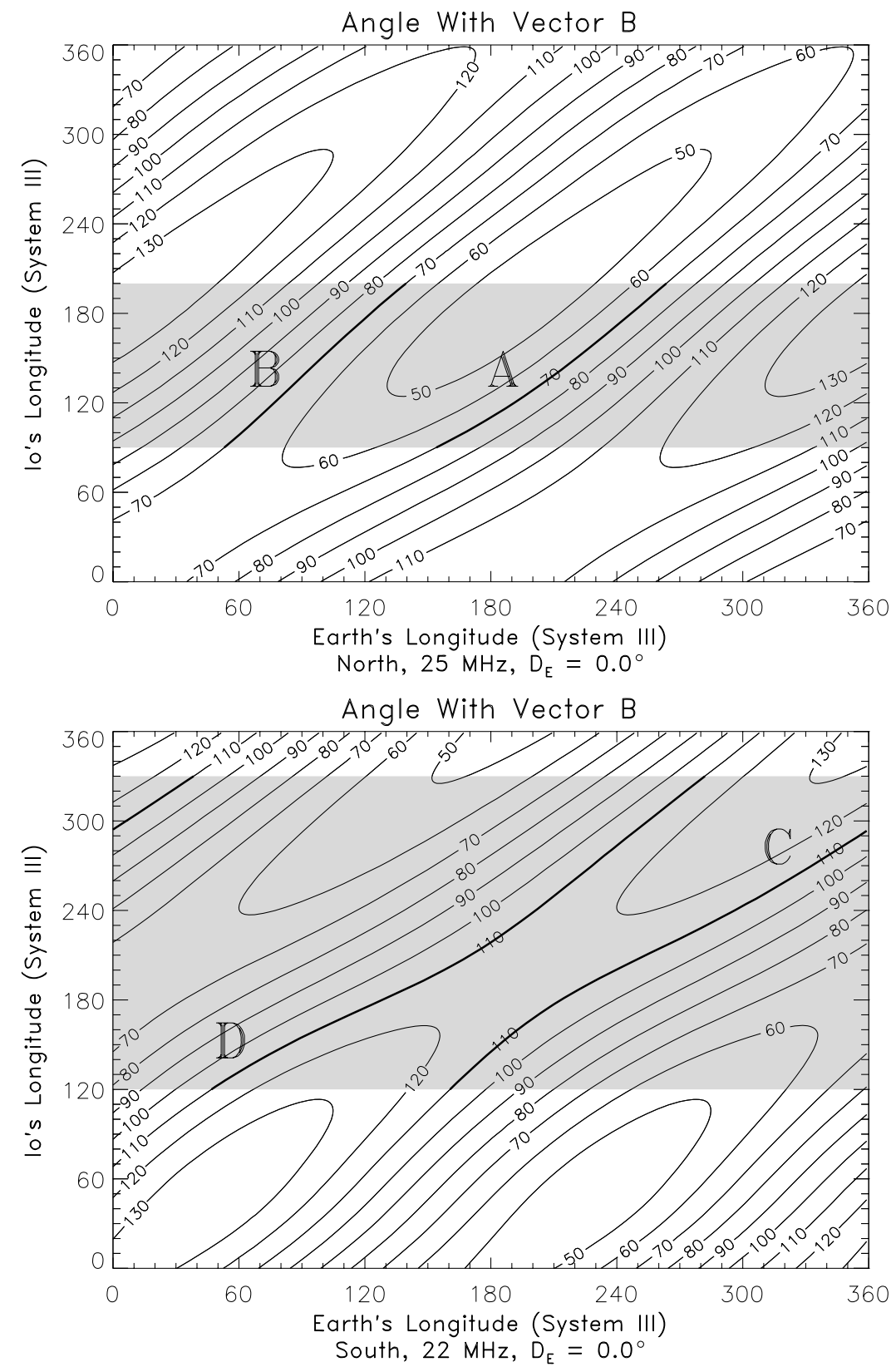

Figure 8. Contours of the angle between the local magnetic field and the direction of the observer (located at Earth). An active longitude range is deduced from the curves in Figure 7 and displayed in gray. The thick segments correspond to emissions with a high probability of occurrence.

frequency. It is important to note that the occurrence "source" regions reported in Figure 1 correspond to DAM events observed with two variable parameters: the frequency between 18 and $32 \mathrm{MHz}$ and $D_{\mathrm{E}}$ in the range from $-3^{\circ}$ to $+3^{\circ}$.

\subsubsection{Sources in the Northern Hemisphere}

\subsubsection{Io-A Source}

[33] For a fixed frequency of about $25 \mathrm{MHz}$, this northern "source" has a CML and a $\Phi_{\mathrm{I}}$ in the intervals $155^{\circ}-265^{\circ}$, and $245^{\circ}-255^{\circ}$, respectively. This modeled position is found, on average, in the region where it is currently observed.

\subsubsection{Io-B Source}

[34] Contrary to the previous "source," the average position of Io-B in $\Phi_{\mathrm{Io}}$ is different from the observed one.
It is found with $\Phi_{\mathrm{Io}}$ values between $117^{\circ}$ and $145^{\circ}$, which corresponds to a shift of about $60^{\circ}$ (at $\mathrm{CML}=90^{\circ}$ ) compared to its classic position. On the other hand, the longitude spreading of Io-B "source" is quasi-similar to the observed one but with a change in the slope $\Delta \mathrm{CML} / \Delta \Phi_{\mathrm{Io}}$ which is found negative in our modeled diagram.

\subsubsection{Sources in the Southern Hemisphere}

\subsubsection{Io-C Source}

[35] The calculation of the CML- $\Phi_{\text {Io }}$ diagram has been done for a fixed frequency of $22 \mathrm{MHz}$. This "source" totally fits the same region as the observed one with a particular feature. It is prolonged toward lower longitude (i.e., between $160^{\circ}$ and $260^{\circ}$ ) which corresponds to a "source" region named Io- $\mathrm{A}^{\prime}$ and discovered by Leblanc 


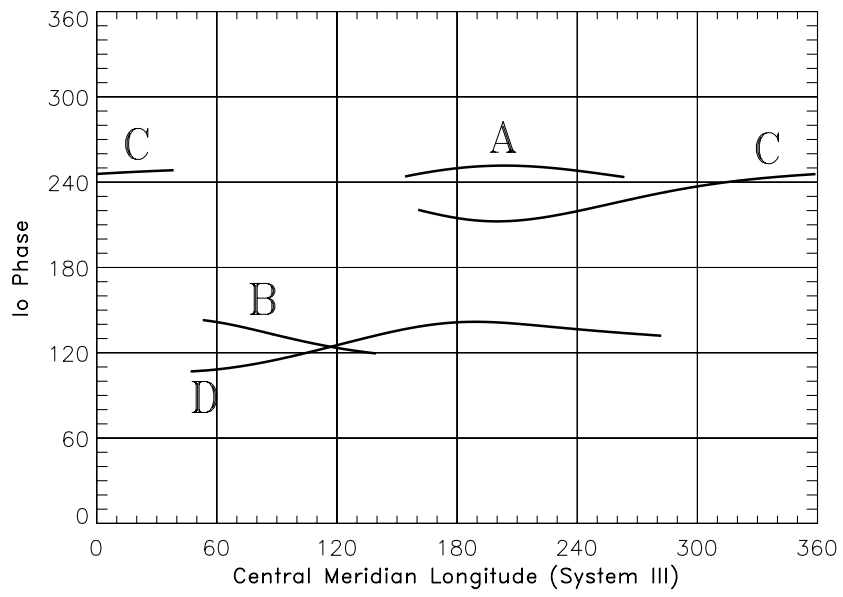

Figure 9. Curves of high occurrence probability versus central meridian longitude and Io phase.

[1981] using Voyager/PRA experiment data. Contrary to the Io-C "source," Io- $\mathrm{A}^{\prime}$ is usually associated to the Northern Hemisphere because of its right-hand polarized component.

\subsubsection{Io-D Source}

[36] It fills the same region as the observed one in longitude and Io phase. However, for the longitude higher than $180^{\circ}$, the modeled Io-D "source" is superposed to the so-called non-Io-A-controlled region. More details about this non-Io-A "source" can be found in the work of Genova and Aubier [1987].

\subsubsection{Summary}

[37] It appears from the previous analysis that the northern "sources" (i.e., Io-A and Io-B) exactly fit the same observed longitudes but the southern ones (i.e., Io-C and Io-D) occupy a wider longitude range. The occurrence in Io phase is found to be similar between the modeled and the observed "sources" except for the Io-B "source" which appears $60^{\circ}$ shifted from its usual position.

\section{Discussion}

[38] The results of our CMI growth rate calculations can be discussed and compared to previous studies with regards to the following points: (1) the basic hypotheses we have chosen for the estimation of the CMI efficiency, in particular the choice of electron distribution function at Io, (2) the common and the different features between our theoretical and the observed CML-Io phase diagrams, and (3) the relationship between the efficiency of the CMI and the observed flux density.

\subsection{Basic Hypotheses}

[39] Among the hypotheses we make, the most decisive one is the choice of the type of electron distribution function since it governs the adjustment of the optimum resonance circle of the CMI and the resulting maximum value of the growth rate $\omega_{i}$ of the waves. So several elements have to be taken into account for a calculation of $\omega_{i}$ at a given frequency.

[40] First of all, we can discuss the choice of initial distribution function at Io. We have chosen a Maxwellian distribution as it is isotropic and depends on only one parameter: its temperature $T$. Such a distribution is probably not realistic and a bi-Maxwellian distribution would allow to take into consideration an anisotropy of the medium. However, a more convenient distribution function exists: the Kappa distribution which behaves like a Maxwellian at low energies and presents a nonthermal tail decreasing with a power law. The Kappa distribution function is well-adapted to space plasmas: Kane et al. [1992] used it to model the hot ions distribution in the jovian magnetodisc, it also accounts for the distribution of electrons in the Io plasma torus measured by Ulysses [Meyer-Vernet et al., 1995; Meyer-Vernet and Moncuquet, 1997].

[41] On the other hand, we suppose that electrons follow an adiabatic motion from Io to Jupiter's ionosphere, where parallel electric fields may exist along the magnetic field lines covered by the electrons. Now observations carried out by the Swedish satellite Viking showed that the AKR sources coincided with acceleration regions and that other possible sources of free energy for the CMI existed in addition to the loss cone: distribution functions with a "hole" in parallel velocity or trapped electron populations at pitch angle $\simeq 90^{\circ}$ [Roux et al., 1993]. Moreover, Louarn et al. [1990] found (still from Viking measurements) that the loss cone distribution was insufficient to produce AKR and that a trapped electron free energy source prevailed over a loss cone one. More recently, observations by the Fast Auroral SnapshoT (FAST) satellite within the AKR source region indicate that the CMI draws its free energy from a "horseshoe" or "shell" electron distribution rather than a loss cone [Ergun et al., 2000, 2001, and references therein]. Such distributions result from electron acceleration by a parallel electric field and appear to be powerful sources for the CMI.

[42] Anyway in the case of Jupiter, on the one hand there is no in situ measurements of the electron distributions within the source regions of the DAM emission; on the other hand the number of electrons precipitating in the Io flux tube might be so great that the loss cone could prevail at that longitude. Nevertheless, the role of a "shell" distribution has to be studied.

[43] Moreover, the electrodynamic interaction of Io with Jupiter's corotating magnetosphere is complicated and has been subject to many studies for a long time. Basing their arguments on a theoretical model developed by Serizawa and Sato [1984] which forecasts the generation of largescale field-aligned potential differences by plasma flows along the mirror field, Erkaev et al. [2001a, 2001b] and Langmayr et al. [2001] suggested that strong pressure pulses could occur along the Io flux tube and produce MHD slow shocks propagating from Io toward Jupiter. According to their model, the location of the ion and electron mirror points at different positions along the Io flux tube would result in a charge separation which would generate a parallel electric field. The latter may affect in a significant way the electron distribution function involved in the CMI mechanism and then it has to be taken into consideration.

\subsection{Comparison Between Occurrence Diagrams}

[44] On the whole, our theoretical model in Figure 9 presents a fairly good agreement with the observed CMI-Io 
phase diagram displayed in Figure 1. However, some features are found to be different, notably: (1) the position of the Io-B "source" is shifted toward higher $\Phi_{\text {Io }}$ values, and (2) the southern "sources" (i.e., Io-C and Io-D) occupy a large longitude range. To understand the origin of these features one needs to report some specific characteristics of the occurrence diagram.

[45] First, it is important to note that the modeled diagram is derived using fixed values of the emitted frequency and jovicentric declination contrary to the observed diagram. Several studies have reported a moving of the occurrence regions [Boudjada and Leblanc, 1992, and references therein] when the jovicentric declination varies from $-3^{\circ}$ to $+3^{\circ}$ over a complete jovian revolution around the Sun which lasts $\sim 12$ years. For instance, in the two extreme cases, i.e., when $D_{\mathrm{E}}$ is equal to $-3^{\circ}$ or $+3^{\circ}$, the DAM emission originates mainly in the Southern or Northern Hemisphere, respectively. Such occurrence diagram features make more difficult the analysis of the exact position of the "source" regions.

[46] The second feature in the occurrence diagram is the dependence on the observed frequency. Several authors have used ground-based and space observations to analyze the variation of the occurrence probability versus the observed frequency. Thieman and Smith [1979] and Genova and Aubier [1985] proved that the "source" areas get wider and wider in CML and $\Phi_{\text {Io }}$ when the frequency decreases from 30 to $15 \mathrm{MHz}$. This means that the occurrence areas overlap, in particular Io-B/Io-D and Io-A/Io-C source regions.

[47] A third characteristic of the CML-Io phase diagram has a connection with the opening angle of the hollow cone. Using geometrical arguments, Thieman and Smith [1979, Figure 7] attempted to explain the occurrence areas of the DAM emission. As shown in their article, some source regions like Io-B are shifted toward higher $\Phi_{\text {Io }}$ when the opening angle is about $70^{\circ}$. This result concurs with our finding in the case of that "source." On the other hand, the extent of the occurrence zones is inversely proportional to the observed frequency which makes some "sources," like Io-C and Io-D, distributed over a larger longitude range. However, Lecacheux et al. [1998] found significant discrepancies between the spectral form associated to Io-controlled emissions and the opening angle derived from the hollow cone model. To explain this discrepancy, the authors considered the presence of refraction effects occurring close to the emission region in Jupiter's magnetosphere which distorted the geometry (in particular the symmetry) of the hollow cone.

\subsection{Growth Rate of the Waves and Observed Radio Flux}

[48] The calculation of the growth rate derived from the optimum resonance circle defined in section 3.7 allows us to study the variation of the CMI efficiency versus the longitude at a given fixed frequency. This one presents a strong modulation at $25 \mathrm{MHz}$ but is nearly constant at $12 \mathrm{MHz}$. In the same time, according to Figure 7, the value of our normalized growth rate increases on average when the frequency varies from $25 \mathrm{MHz}$ to $12 \mathrm{MHz}$. Our study concerns the calculation of the growth rate of the waves that we suppose to be representative of the occurrence probability of the emission. Nevertheless this growth rate does not correspond exactly to the flux density of the radiation escaping from the planet. Actually such a calculation of flux density requires the knowledge of the precise source size; on needs to know either if the growth of the wave is linear or if a saturation of the mechanism intervenes; finally one would have to solve a transfer equation too.

[49] Until today, all the previous studies of the DAM occurrence versus the longitude and the Io phase have never reported any average value of the observed flux associated to each event in the CML-Io phase diagram. This task is very difficult because of its dependence on several parameters: the spectral index necessary to convert flux densities into power which is poorly known, the reception effective area and the corresponding antenna beam which is usually not automatically analyzed, observation conditions due to Earth's ionosphere effect which vary with locations, hours and seasons. Because of the previous observation features, there is a division between the study of the DAM occurrence probability and the measurement of the observed flux.

[50] Carr et al. [1983] estimated the DAM power to $6 \times$ $10^{9} \mathrm{~W}$ at $10 \mathrm{MHz}$ on condition that the emission occurs in a thin hollow cone. Using Voyager/PRA experiment data, Alexander et al. [1981] reported the variation of the average flux density and the occurrence probability versus the central meridian longitude. In the work of Alexander et al. [1981, Figure 9] one can see that the average flux density is $\sim 2 \times 10^{-21} \mathrm{Wm}^{-2} \mathrm{~Hz}^{-1}$ and $\sim 6 \times 10^{-23} \mathrm{Wm}^{-2} \mathrm{~Hz}^{-1}$ at $4 \mathrm{AU}$ when the observed frequency is $\sim 12 \mathrm{MHz}$ and $\sim 25 \mathrm{MHz}$, respectively. This suggests that the observed flux density decreases by about two orders of magnitude from 25 to $12 \mathrm{MHz}$. This noticing is compatible with our calculation of the growth rate at low and high frequencies: the ratio of the flux densities is $S^{\left.(25 \mathrm{MHz}) / S^{(12} \mathrm{MHz}\right)} \sim 0.03$ while $\omega_{i}^{(25 \mathrm{MHz})} / \omega_{i}^{(12 \mathrm{MHz})} \sim 0.3$.

\section{Conclusion}

[51] A variety of theories has been proposed to explain the jovian decametric emission since its discovery in 1955. The cyclotron maser instability thought up by $W u$ and Lee [1979] has proved to be the best candidate to account for Earth's auroral kilometric emissions [Grabbe, 1981], and also for the jovian decametric radiation [Goldstein and Goertz, 1983]. However, part of the jovian decametric phenomenology (e.g., spectral features, active longitudes, elliptic polarization, etc.) is still subject to discussions.

[52] In this paper, we have focused on the dependence of the DAM emission on the planetary rotation which is a wellknown feature of the occurrence of this radiation. Using simple hypotheses we have studied the growth rate of the CMI mechanism (associated to a loss cone distribution function) as a function of several jovian DAM key parameters, like the observed frequency, the central meridian longitude (i.e., the observer's longitude) and the phase of the satellite Io. It emerges from our study that there exists an "active" longitude range in the northern and southern jovian hemispheres which favors the DAM emission. The modeled and the observed occurrence diagram of DAM emissions are found to be fairly similar, in particular with 
regard to the CML parameter. This result is very motivating considering the simple set of hypotheses. A more elaborate analysis is in preparation; it will concern several ingredients of our model which are subject to discussion, in particular: the type of electron distribution function, the opening angle and the location of the beaming cone.

\section{Appendix A: Linear Growth Rate of the CMI in Homogeneous Medium}

[53] In the following, we present the calculations allowing to derive the growth rate and the resonance conditions of the cyclotron maser instability in a homogeneous medium. These calculations are inspired by Le Quéau et al. [1984a, 1984b, and references therein].

\section{A1. Basic Equations}

[54] Let us consider a magnetized plasma in which electromagnetic waves can develop. The basis equations are naturally Maxwell's equations:

$$
\begin{gathered}
\operatorname{div} \mathbf{E}=\frac{\rho}{\varepsilon_{0}} \\
\operatorname{curl} \mathbf{E}=-\frac{\partial \mathbf{B}}{\partial t} \\
\operatorname{div} \mathbf{B}=0 \\
\operatorname{curl} \mathbf{B}=\mu_{0} \mathbf{j}+\varepsilon_{0} \mu_{0} \frac{\partial \mathbf{E}}{\partial \mathbf{t}}
\end{gathered}
$$

and Vlasov's equations for all ions species and electrons which are present in the magnetized plasma:

$$
\frac{\partial f_{\alpha}}{\partial t}+\mathbf{v} \cdot \frac{\partial f_{\alpha}}{\partial \mathbf{r}}+\mathbf{F} \cdot \frac{\partial f_{\alpha}}{\partial \mathbf{p}}=0
$$

For each species characterized by its index $\alpha$, the distribution function $f_{\alpha}(t, \mathbf{r}, \mathbf{p})$ is defined in order that $d^{6} n=f_{\alpha}(t, \mathbf{r}, \mathbf{p}) d^{3} \mathbf{r} d^{3} \mathbf{p}$ is the number of ions or electrons contained in a box of size $d^{3} \mathbf{r} d^{3} \mathbf{p}$ in the phase space at $(\mathbf{r}, \mathbf{p})$.

[55] The charge and current densities are:

$$
\begin{aligned}
\rho & =\iiint \sum_{\alpha} q_{\alpha} f_{\alpha}(t, \mathbf{r}, \mathbf{p}) d^{3} \mathbf{p} \\
\mathbf{j} & =\iiint \sum_{\alpha} q_{\alpha} f_{\alpha}(t, \mathbf{r}, \mathbf{p}) \mathbf{v} d^{3} \mathbf{p} .
\end{aligned}
$$

[56] The above equations are linearized to the first order: $f_{\alpha}=f_{\alpha}^{(0)}+f_{\alpha}^{(1)}, \rho_{\alpha}=\rho_{\alpha}^{(0)}+\rho_{\alpha}^{(1)}$.

\section{A2. Zeroth Order}

[57] The medium is supposed to be homogeneous and standing. It is submerged by a uniform magnetic field $\mathbf{B}_{0}$. In the following, the cylindrical coordinates $\left(p_{\|}, p_{\perp}, \varphi\right)$ are used in the momentum space, the reference axis is parallel to $\mathbf{B}_{0}$ so that:

$$
\begin{gathered}
p_{x}=p_{\perp} \cos \varphi \\
p_{y}=p_{\perp} \sin \varphi \\
p_{z}=p_{\|} .
\end{gathered}
$$

[58] Vlasov equation (A5) implies at zeroth order:

$$
\frac{f_{\alpha}^{(0)}}{\partial \varphi}=0
$$

which means symmetry round $\mathbf{B}_{0}$, i.e.,:

$$
f_{\alpha}^{(0)}(\mathbf{r}, \mathbf{p})=f_{\alpha}^{(0)}\left(p_{\|}, p_{\perp}\right)
$$

\section{A3. First Order}

[59] Equation (A5) can be rewritten at first order:

$$
\begin{gathered}
\frac{\partial f_{\alpha}^{(1)}}{\partial t}+\mathbf{v} \cdot \frac{\partial f_{\alpha}^{(1)}}{\partial \mathbf{r}}+q_{\alpha}\left(\mathbf{v} \times \mathbf{B}_{0}+\mathbf{E}^{(1)}+\mathbf{v} \times \mathbf{B}^{(1)}\right) \\
\cdot\left[\frac{\partial f_{\alpha}^{(0)}}{\partial \mathbf{p}}+\frac{\partial f_{\alpha}^{(1)}}{\partial \mathbf{p}}\right]=0 .
\end{gathered}
$$

[60] Now we can make the assumption that $\mathbf{E}^{(1)}, \mathbf{B}^{(1)}$ and $f_{\alpha}^{(1)}$ express a wave perturbation proportional to $\exp [i(\mathbf{k} \cdot \mathbf{r}$ $-\omega t)$ with $\omega \in \mathbf{C}$ and $\mathbf{k} \in \mathbf{R}^{3}$. We also assume that only $f_{e}^{(1)}$ (electron distribution function) contributes to $\rho^{(1)}$ and $\mathbf{j}^{(1)}$ and we note $f$ instead of $f_{e}$. Thus equation (A12) becomes:

$$
i(\mathbf{k} \cdot \mathbf{p}-\gamma m \omega) f^{(1)}+m \omega_{c} \frac{\partial f^{(1)}}{\partial \varphi}=\gamma m e \mathbf{E}^{(1)} \cdot \frac{\partial f^{(0)}}{\partial \mathbf{p}}
$$

with $\omega_{c}=e B_{0} / m ; e$ and $m$ being respectively the charge and mass of electron and $\gamma$ referring to the Lorentz factor. The following notations are introduced:

$$
\begin{gathered}
E_{ \pm}=E_{x} \pm i E_{y} \\
E_{\|}=E_{z},
\end{gathered}
$$

the $z$ axis being parallel to $\mathbf{B}_{0}$. With those notations, a real $E_{-}$or $E_{+}$denotes a circular right- or left-handed electric field, respectively. The same notations will be used for $\mathbf{j}^{(1)}$ and $\mathbf{v}$. Finally equation (A13) becomes:

$$
\begin{aligned}
i(\mathbf{k} \cdot \mathbf{p}-\gamma m \omega) f^{(1)}+m \omega_{c} \frac{\partial f^{(1)}}{\partial \varphi}= & \frac{\gamma m e}{2}\left(E_{+}^{(1)} e^{-i \varphi}+E_{-}^{(1)} e^{i \varphi}\right) \frac{\partial f^{(0)}}{\partial p_{\perp}} \\
& +\gamma m e E_{\|}^{(1)} \frac{\partial f^{(0)}}{\partial p_{\|}} . \quad(\mathrm{A} 15)
\end{aligned}
$$


[61] Equation (A14) is a linear differential equation in with a second member which can be easily integrated by classical methods:

$\begin{aligned} f^{(1)}(t, \mathbf{r}, \mathbf{p})= & \frac{\gamma m e}{2 i \pi} e^{-i k_{\perp} p_{\perp} \sin \varphi / m \omega_{c}} \times \sum_{n \in \mathbf{Z}} \frac{e^{i n \varphi}}{k_{\|} p_{\|}-\gamma m \omega+n m \omega_{c}} \\ & \times\left\{\frac{E_{+}^{(1)}}{2} \frac{\partial f^{(0)}}{\partial p_{\perp}} J_{n+1}+\frac{E_{-}^{(1)}}{2} \frac{\partial f^{(0)}}{\partial p_{\perp}} J_{n-1}+E_{\|}^{(1)} \frac{\partial f^{(0)}}{\partial p_{\|}} J_{n}\right\},\end{aligned}$

$J_{n}=J_{n}\left(k_{\perp} p_{\perp} / m \omega_{c}\right)$ is a Bessel function of integer-order, $k_{\perp}$ and $k_{\|}$are the wave vector perpendicular and parallel to $\mathbf{B}_{0}$, respectively.

[62] Thus the charge and current densities are (at the first order):

$$
\begin{gathered}
\rho^{(1)}(t, \mathbf{r})=\iiint-e f^{(1)}(t, r, p) p_{\perp} d p_{\|} d p_{\perp} d \varphi \\
\mathbf{j}^{(1)}(t, \mathbf{r})=\iiint-e f^{(1)}(t, \mathbf{r}, \mathbf{p}) \mathbf{v} p_{\perp} d p_{\|} d p_{\perp} d \varphi,
\end{gathered}
$$

with $p_{\|} \in \mathbf{R}, p_{\perp} \in \mathbf{R}_{+}$and $\varphi \in[0,2 \pi]$.

[63] Replacing in equations (A17) and (A18) $f^{(1)}(t, \mathbf{r}, \mathbf{p})$ by its expression given in (A16), then making the integration over $\varphi$, one obtains an expansion for $\rho^{(1)}, j_{ \pm}^{(1)}=j_{x}^{(1)} \pm i j_{y}^{(1)}$ and $j_{\|}^{(1)}=j_{z}^{(1)}$ as a series the terms of which contain products of Bessel functions like $J_{n}\left(k_{\perp} p_{\perp} / m \omega_{c}\right) \cdot J_{n^{\prime}}\left(k_{\perp p \perp} / m \omega_{c}\right)$. The result can be simplified significantly if one takes into account only nonnegligible terms in $J_{0}^{2}$. Assuming $k_{\perp} p_{\perp} / m \omega_{c} \ll 1$ on the major part of $f^{(0)}\left(p_{\|}, p_{\perp}\right)$, one may make the approximation $J_{0}^{2}\left(k_{\perp} p_{\perp} / m \omega_{c}\right) \sim 1$. Finally, one obtains:

$$
\begin{gathered}
\rho^{(1)}(t, \mathbf{r})=E_{\|}^{(1)} \frac{e^{2}}{2 i \pi} \iint_{\mathcal{D}} \frac{\frac{\partial f^{(0)}}{\partial p_{\|}}}{\gamma m \omega-k_{\|} p_{\|}} \gamma m p_{\perp} d p_{\|} d p_{\perp} \\
j_{ \pm}^{(1)}(t, \mathbf{r})=\frac{E_{ \pm}^{(1)}}{2} \frac{e^{2}}{2 i \pi} \iint_{\mathcal{D}} \frac{\frac{\partial f^{(0)}}{\partial p_{\perp}}}{\gamma m \omega-k_{\|} p_{\|} \pm m \omega_{c}} p_{\perp}^{2} d p_{\|} d p_{\perp} \\
j_{\|}^{(1)}(t, \mathbf{r})=E_{\|}^{(1)} \frac{e^{2}}{2 i \pi} \iint_{\mathcal{D}} \frac{\frac{\partial f^{(0)}}{\partial p_{\|}}}{\gamma m \omega-k_{\|} p_{\|}} p_{\|} p_{\perp} d p_{\|} d p_{\perp},
\end{gathered}
$$

where the integration domain is $\mathcal{D}=\left\{\left(p_{\|}, p_{\perp}\right) \in \mathbf{R} \times \mathbf{R}_{+}\right\}$.

[64] The terms of first order in Maxwell equations allow us to derive the electric field of the waves $\mathbf{E}^{(1)}$ as a function of $\rho^{(1)}$ and $\mathbf{j}^{(1)}$ :

$$
\left(k^{2}-\frac{\omega^{2}}{c^{2}}\right) \mathbf{E}^{(1)}+i \mu_{0} c^{2} \rho^{(1)} \mathbf{k}-i \mu_{0} \omega \mathbf{j}^{(1)}=\mathbf{0} .
$$

[65] Replacing $\rho^{(1)}$ and $\mathbf{j}^{(1)}$ by their expressions given by (A19), (A20) and (A21), one can rewrite (A22) in a matrix form:

$$
D(\mathbf{k}, \omega) \cdot\left(\begin{array}{c}
E_{+}^{(1)} \\
E_{-}^{(1)} \\
E_{\|}^{(1)}
\end{array}\right)=\left(\begin{array}{l}
0 \\
0 \\
0
\end{array}\right)
$$

where $D(\mathbf{k}, \omega)$ is a square matrix:

$$
D(\mathbf{k}, \omega)=\left(\begin{array}{llr}
\frac{K}{2}-\frac{i \mu_{0} \omega}{4} J_{+} & \frac{K}{2}-\frac{i \mu_{0} \omega}{4} J_{-} & i \mu_{0} c^{2} k_{\perp} R \\
\frac{K}{2 i}-\frac{\mu_{0} \omega}{4} J_{+} & -\frac{K}{2 i}+\frac{\mu_{0} \omega}{4} J_{-} & 0 \\
0 & 0 & K+i \mu_{0} c^{2} k_{\|} R-i \mu_{0} \omega J_{\|}
\end{array}\right)
$$

with

$$
K=\left(k^{2}-\frac{\omega^{2}}{c^{2}}\right)
$$

and

$$
R=\frac{e^{2}}{2 i \pi} \iint_{\mathcal{D}} \frac{\frac{\partial f^{(0)}}{\partial p_{\|}}}{\gamma m \omega-k_{\|} p_{\|}} \gamma m p_{\perp} d p_{\|} d p_{\perp}
$$

$$
\begin{gathered}
J_{ \pm}=\frac{e^{2}}{2 i \pi} \iint_{\mathcal{D}} \frac{\frac{\partial f^{(0)}}{\partial p_{\perp}}}{\gamma m \omega-k_{\|} p_{\|} \pm m \omega_{c}} p_{\perp}^{2} d p_{\|} d p_{\perp} \\
J_{\|}=\frac{e^{2}}{2 i \pi} \iint_{\mathcal{D}} \frac{\frac{\partial f^{(0)}}{\partial p_{\|}}}{\gamma m \omega-k_{\|} p_{\|}} p_{\|} p_{\perp} d p_{\|} d p_{\perp} .
\end{gathered}
$$

[66] The general dispersion equation of the waves is obtained by setting the determinant of the matrix $D(\mathbf{k}, \omega)$ equal to zero. In the case we are studying, we only consider the growth rate of right-handed circular waves which have a stronger interaction with the electron population the motion of which tends to a right-handed helix. Thus one sets $E_{+}^{(1)}=$ $E_{\|}^{(1)}=0$ in equation (A23), so that the equation of dispersion writes:

$$
\left(k^{2}-\frac{\omega^{2}}{c^{2}}\right)-\frac{\mu_{0} e^{2} \omega}{4 \pi} \iint_{\mathcal{D}} \frac{\frac{\partial f^{(0)}}{\partial p_{\perp}}}{\gamma m \omega-k_{\|} p_{\|}-m \omega_{c}} p_{\perp}^{2} d p_{\|} d p_{\perp}=0 .
$$

[67] The electron distribution taken into account here is assumed to be non relativistic so that one may set $\mathbf{p}=m \mathbf{v}$ or $\gamma=1$ everywhere except in the denominator where $\omega \simeq \omega_{c}$. One can also introduce the distribution function in velocity:

$$
f\left(v_{\|}, v_{\perp}\right)=m^{3} f^{(0)}\left(p_{\|}, p_{\perp}\right),
$$

so that the equation of dispersion (A28) becomes:

$$
k^{2}-\frac{\omega^{2}}{c^{2}}-\frac{\mu_{0} e^{2} \omega}{4 \pi m} \iint_{\mathcal{D}} \frac{\partial f / \partial v_{\perp}}{\omega-k_{\|} v_{\|}-\frac{\omega_{c}}{\gamma}} v_{\perp}^{2} d v_{\|} d v_{\perp}=0
$$

with $\omega=\omega_{r}+i \omega_{i} \in \mathbf{C}$. The imaginary part of the pulsation $\omega_{i}$ represents the growth rate of the waves $\left(\omega_{i}>0\right.$ corresponds to an instability).

[68] At this stage of the calculation, it is relevant to apply Plemelj's formula to (A31). (In Plemelj's formula (A31), the 
symbol $\mathrm{P}$ denotes the Cauchy principal value defined as: $\left.\mathrm{P} \int_{-\infty}^{+\infty} \frac{f(x)}{x-x_{0}} d x=\lim _{\varepsilon \rightarrow 0^{+}}\left[\int_{-\infty}^{\mathrm{x}_{0}-\varepsilon} \frac{f(x)}{x-x_{0}} d x+\int_{x_{0}+\varepsilon}^{+\infty} \frac{f(x)}{x-x_{0}} d x\right].\right)$

$$
\lim _{\varepsilon \rightarrow 0^{+}} \int_{0}^{+\infty} \frac{f(x)}{x-x_{0} \mp i \varepsilon} d x=\mathrm{P} \int_{-\infty}^{+\infty} \frac{f(x)}{x-x_{0}} d x \pm i \pi f\left(x_{0}\right),
$$

which allows us to derive the growth rate of the unstable waves (assuming that $\left|\omega_{i}\right| \ll \omega_{r}$ ):

$$
\omega_{i}=\frac{e^{2}}{8 \varepsilon_{0} m} \int_{0}^{+\infty} v_{\perp}^{2} d v_{\perp} \int_{-\infty}^{+\infty} \frac{\partial f}{\partial v_{\perp}} \delta\left(\omega_{r}-k_{\|} v_{\|}-\frac{\omega_{c}}{\gamma}\right) d v_{\|}
$$

where $\delta$ is here Dirac's distribution.

[69] Normalizing the distribution function $f\left(v_{\|}, v_{\perp}\right)$ to unity and introducing the plasma frequency, equation (A32) rewrites:

$$
\omega_{i}=\frac{\omega_{p}^{2}}{8} \int_{0}^{+\infty} v_{\perp}^{2} d v_{\perp} \int_{-\infty}^{+\infty} \frac{\partial f}{\partial v_{\perp}} \delta\left(\omega_{r}-k_{\|} v_{\|}-\frac{\omega_{c}}{\gamma}\right) d v_{\|}
$$

with $\omega_{p}^{2}=n e^{2} / m \varepsilon_{0}, n$ denoting the electron density, and

$$
\iiint f\left(v_{\|}, v_{\perp}\right) d^{3} \mathbf{v}=1
$$

Equation (A33) is nothing less than the fundamental result obtained by $W u$ and Lee [1979].

[70] Acknowledgments. Arthur Richmond thanks Michael Kaiser for his assistance in evaluating this paper.

\section{References}

Alexander, J. K., T. D. Carr, J. R. Thieman, J. J. Schauble, and A. C. Riddle (1981), Synoptic observations of Jupiter's radio emissions: Average statistical properties observed by Voyager, J. Geophys. Res., 86, 85298545.

Aubier, A., M. Y. Boudjada, P. Moreau, P. H. M. Galopeau, A. Lecacheux, and H. O. Rucker (2000), Statistical studies of jovian decameter emissions observed during the same period by Nancay Decameter Array (France) and WAVES experiment aboard Wind spacecraft, Astron. Astrophys., 354, 1101-1109.

Bigg, E. K. (1964), Influence of the satellite Io on Jupiter's decametric emission, Nature, 203, 1008-1011.

Boudjada, M. Y., and Y. Leblanc (1992), The variability of jovian decametric radiation from 1978 to 1988, Adv. Space Res., 12(8), 95-98, doi:10.1016/0273-1177(92)90382-8.

Burke, B. F., and K. L. Franklin (1955), Observations of a variable radio source associated with planet Jupiter, J. Geophys. Res., 60, 213-217.

Carr, T. D., and M. D. Desch (1976), Recent decametric and hectometric observations of Jupiter, in IAU Colloquium 30: Jupiter: Studies of the Interior, Atmosphere, Magnetosphere and Satellites, pp. 693-737, Univ. of Ariz. Press, Tucson.

Carr, T. D., M. D. Desch, and J. K. Alexander (1983), Phenomenology of magnetospheric radio emissions, in Physics of the Jovian Magnetosphere, edited by A. J. Dessler, pp. 226-284, Cambridge Univ. Press, New York.

Connerney, J. E. P. (1992), Doing more with Jupiter's magnetic field, in Planetary Radio Emissions III, edited by H. O. Rucker, S. J. Bauer, and M. L. Kaiser, pp. 13-33, Aust. Acad. of Sci. Press, Vienna.

Connerney, J. E. P. (1993), Magnetic fields of the outer planets, J. Geophys. Res., 98, 18,659-18,679.

Dulk, G. A. (1965a), Io-related radio emission from Jupiter, Science, 148, $1585-1589$.

Dulk, G. A. (1965b), Io-related radio emission from Jupiter, Ph.D. thesis, Univ. of Colo., Boulder.

Ellis, G. R. A. (1965), The decametric radio emissions of Jupiter, Radio Sci., 69D, 1513-1530.
Ergun, R. E., C. W. Carlson, J. P. McFadden, G. T. Delory, R. J. Strangeway, and P. L. Pritchett (2000), Electron-cyclotron maser driven by chargedparticle acceleration from magnetic field-aligned electric fields, Astrophys. J., 538, 456-466.

Ergun, R. E., Y.-J. Su, and F. Bagenal (2001), Terrestrial radio emission: AKR, in Planetary Radio Emissions $V$, edited by H. O. Rucker, M. L. Kaiser, and Y. Leblanc, pp. 271-280, Aust. Acad. of Sci. Press, Vienna.

Erkaev, N. V., V. S. Semenov, V. A. Shaidurov, D. Langmayr, H. K. Biernat, and H. O. Rucker (2001a), Effects of MHD slow shocks propagating along the Io flux tube, in Planetary Radio Emissions $V$, edited by H. O. Rucker, M. L. Kaiser, and Y. Leblanc, pp. 389-394, Aust. Acad. of Sci. Press, Vienna.

Erkaev, N. V., V. S. Semenov, V. A. Shaidurov, D. Langmayr, H. K. Biernat, and H. O. Rucker (2001b), Propagation of nonlinear slow waves produced by pressure pulses along the Io flux tube, Adv. Space Res., 28(10), 1481-1488, doi:10.1016/S0273-1177(01)0,550-6.

Eshleman, V. R., G. L. Tyler, G. E. Wood, G. F. Lindal, J. D. Anderson, G. S. Levy, and T. A. Croft (1979a), Radio science with Voyager 1 at Jupiter-Preliminary profiles of the atmosphere and ionosphere, Science, 204, 976-978.

Eshleman, V. R., G. L. Tyler, G. E. Wood, G. F. Lindal, J. D. Anderson, G. S. Levy, and T. A. Croft (1979b), Radio science with Voyager at Jupiter-Initial Voyager 2 results and a Voyager 1 measure of the Io torus, Science, 206, 959-962.

Genova, F., and M. G. Aubier (1985), Io-dependent sources of the jovian decameter emission, Astron. Astrophys., 150, 139-150.

Genova, F., and M. G. Aubier (1987), High frequency limit and visibility of the non-Io and Io-dependent jovian decameter radio emission, Astron. Astrophys., 177, 303-309.

Genova, F., P. Zarka, and A. Lecacheux (1989), Jupiter decametric radiation, in Time Variable Phenomena in the Jovian System, Rep. NASA SP-494, edited by M. J. S. Belton, R. A. West, and J. Rahe, pp. 156-174, NASA, Greenbelt, Md.

Goldstein, M. L., and C. K. Goertz (1983), Theories of radio emissions and plasma waves, in Physics of the Jovian Magnetosphere, edited by A. J. Dessler, pp. 317-352, Cambridge Univ. Press, New York.

Grabbe, C. L. (1981), Auroral kilometric radiation: A theoretical review, Rev. Geophys., 19, 627-633.

Hanasz, J., M. Y. Boudjada, R. Schreiber, Z. Krawczyk, M. Malycha, M. M. Mogilevsky, H. O. Rucker, and T. V. Romantsova (2000), Dynamic spectra of the Stokes parameters for the dayside and nightside Auroral Kilometric Radiation, Geophys. Res. Lett., 27, 1631-1634.

Kane, M., B. H. Mauk, E. P. Keath, and S. M. Krimigis (1992), A convected kappa distribution model for hot ions in the jovian magnetodisc, Geophys. Res. Lett., 19, 1435-1438.

Ladreiter, H. P. (1991), The cyclotron maser instability: Application to low-density magnetoplasmas, Astrophys. J., 370, 419-426.

Langmayr, D., N. V. Erkaev, V. S. Semenov, V. A. Shaidurov, H. K. Biernat, H. O. Rucker, D. F. Vogl, and S. Mühlbachler (2001), Field-aligned electric field in the Io flux tube as a result of a pressure pulse near Io, in Planetary Radio Emissions V, edited by H. O. Rucker, M. L. Kaiser, and Y. Leblanc, pp. 375-380, Aust. Acad. of Sci. Press, Vienna.

Leblanc, Y. (1981), On the arc structure of the DAM Jupiter emission, J. Geophys. Res., 86, 8546-8560.

Leblanc, Y., A. Gerbault, L. Denis, and A. Lecacheux (1993), A catalogue of jovian decametric radio observations from January 1988 to December 1990, Astron. Astrophys., 98(suppl.), 529-546.

Leblanc, Y., G. A. Dulk, and F. Bagenal (1994), On Io's excitation and the origin of Jupiter's decametric radiation, Astron. Astrophys., 290, 660673 .

Lecacheux, A., A. Boischot, M. Y. Boudjada, and G. A. Dulk (1991), Spectra and complete polarization state of two, Io-related, radio storms from Jupiter, Astron. Astrophys., 251, 339-348.

Lecacheux, A., M. Y. Boudjada, H. O. Rucker, J. L. Bougeret, R. Manning, and M. L. Kaiser (1998), Jovian decameter emissions observed by the Wind/WAVES radioastronomy experiment, Astron. Astrophys., 329, $776-784$.

Lee, L. C., and C. S. Wu (1980), Amplification of radiation near cyclotron frequency due to electron population inversion, Phys. Fluids, 23, 13481354.

Le Quéau, D., R. Pellat, and A. Roux (1984a), Direct generation of the auroral kilometric radiation by the maser synchrotron instability: An analytical approach, Phys. Fluids, 27, 247-265.

Le Quéau, D., R. Pellat, and A. Roux (1984b), Direct generation of the auroral kilometric radiation by the maser synchrotron instability: Physical mechanism and parametric study, J. Geophys. Res., 89, $2831-2841$

Louarn, P., A. Roux, H. de Féraudy, D. Le Quéau, and M. L. André M. (1990), Trapped electrons as a free energy source for the auroral kilometric radiation, J. Geophys. Res., 95, 5983-5995. 
Melrose, D. B. (1976), An interpretation of Jupiter's decametric radiation and the terrestrial kilometric radiation as direct amplified gyroemission, Astrophys. J., 207, 651-662.

Meyer-Vernet, N., and M. Moncuquet (1997), The structure of the Io plasma torus after Ulysses encounter, in Planetary Radio Emissions IV, edited by H. O. Rucker, S. J. Bauer, and A. Lecacheux, pp. 167-179, Aust. Acad. Sci. Press, Vienna.

Meyer-Vernet, N., M. Moncuquet, and S. Hoang (1995), Temperature inversion in the Io plasma torus, Icarus, 116, 202-213, doi:10.1006/ icar.1995.1121.

Omidi, N., and D. A. Gurnett (1982), Growth rate calculations of auroral kilometric radiation using the relativistic resonance condition, J. Geophys. Res., 87, 2377-2383.

Omidi, N., C. S. Wu, and D. A. Gurnett (1984), Generation of auroral kilometric and $\mathrm{Z}$ mode radiation by the cyclotron maser mechanism, J. Geophys. Res., 89, 883-895.

Roux, A., A. Hilgers, H. de Féraudy, D. Le Quéau, P. Louarn, S. Perraut, A. Bahnsen, M. Jespersen, E. Ungstrup, and M. André (1993), Auroral kilometric radiation sources: In situ and remote observations from Viking, J. Geophys. Res., 98, 11,657-11,670.

Serizawa, Y., and T. Sato (1984), Generation of large scale potential difference by currentless plasma jets along the mirror field, Geophys. Res. Lett., 11, 595-598.

Smith, R. A. (1976), Models of jovian decametric radiation, in IAU Colloquium 30: Jupiter: Studies of the Interior, Atmosphere, Magnetosphere and Satellites, edited by T. Gehrels, pp. 1146-1189, Univ. of Ariz. Press, Tucson.
Thieman, J. R. (1979), A catalog of jovian decameter-wave radio observations from 1957-1978, NASA STI/Recon Tech. Rep. N, 79, $34,137$.

Thieman, J. R., and A. G. Smith (1979), Detailed geometrical modeling of Jupiter's Io-related decametric radiation, J. Geophys. Res., 84, 26662674.

Warwick, J. W., G. A. Dulk, and A. C. Riddle (1975), Jupiter radio emission: January 1960 to March 1975, Tech. Rep., Radio Astron. Obs., Univ. of Colo., Boulder.

Wu, C. S., and H. P. Freund (1977), Induced emission of Jupiter's decametric radiation by Io-accelerated electrons, Astrophys. J., 213, $575-587$.

Wu, C. S., and L. C. Lee (1979), A theory of the terrestrial kilometric radiation, Astrophys. J., 230, 621-626.

Wu, C. S., H. K. Wong, D. J. Gorney, and L. C. Lee (1982), Generation of the auroral kilometric radiation, J. Geophys. Res., 87, 4476-4487.

M. Y. Boudjada and H. O. Rucker, Abteilung für Physik des erdnahen Weltraums, OAW-Forschungszentrum Graz, Schmiedlstraße 6, A-8042 Graz, Austria. (mohammed.boudjada@oeaw.ac.at; helmut.rucker@oeaw. ac.at)

P. H. M. Galopeau, Centre d'Étude des Environnements Terrestre et Planétaires, 10-12 Avenue de l'Europe, F-78140 Vélizy, France. (patrick. galopeau@cetp.ipsl.fr) 